\title{
Contracepção hormonal e anti-retrovirais em mulheres infectadas pelo HIV
}

\author{
Hormonal contraception and antiretroviral therapy among HIV-infected women
}

\author{
Eliana Amaral ${ }^{1}$, Marco Aurélio Martino Viscola ${ }^{2}$, Luis Bahamondes ${ }^{3}$
}

\section{Resumo}

Há controvérsia sobre a relação entre o uso de contraceptivos hormonais e o risco de adquirir o vírus da imunodeficiência humana (HIV), e pouco se sabe sobre os efeitos da contracepção hormonal em mulheres infectadas (efeitos colaterais, distúrbios menstruais, progressão da doença, interações com terapias anti-retrovirais). O objetivo deste artigo foi revisar os dados disponíveis quanto à vulnerabilidade ao HIV e à sua transmissibilidade na vigência do uso de contraceptivos hormonais bem como as conseqüências potenciais do uso desses contraceptivos por mulheres HIV-positivas sob terapia anti-retroviral (TARV), com ênfase nas interações medicamentosas. Concluiu-se que ainda não é possível elaborar recomendações, baseadas em evidências, sobre a contracepção hormonal em mulheres portadoras do HIV sob TARV. Assim, os infectologistas e os ginecologistas devem estar atentos às interações potenciais que possam representar aumento de efeitos adversos, individualizando a orientação sobre os esteróides contraceptivos, suas doses e vias de administração, considerando a TARV em uso.

PALAVRAS-CHAVES: Infecções por HIV; HIV; Terapia anti-retroviral de alta atividade; Anticoncepção; Anticoncepcionais orais hormonais

\section{Abstract}

There is much controversy regarding the realtionship between the use of hormonal contraceptives and the risk of acquiring human immunodeficiency virus (HIV), and little is known about the effects of hormonal contraception in HIV-infected women (adverse events, menstrual disorders, disease progression, antiretroviral therapy interactions). The aim of the present study was to review available data regarding HIV vulnerability and transmission associated with hormonal contraceptives and the use of these contraceptives by women on antiretroviral therapy, with emphasis on drug interactions. In conclusion, it was not possible to offer evidence-based recommendations for the use of hormonal contraceptives among HIV-infected women under antiretroviral therapy. Infectious disease specialists and gynecologists providing care should be cautious about potential drug interaction leading to increase in adverse events, individualizing contraceptive drugs, route, and dosage, according to the antiretroviral therapy under use.

KEYWORDS: HIV infections; HIV; Antiretroviral therapy, highly active; Contraception; Contraceptives, oral, hormonal

1 Professora-Assistente do Departamento de Tocoginecologia da Faculdade de Ciências Médicas da Universidade Estadual de Campinas - UNICAMP; Diretora da Divisão de Obstetrícia do CAISM/UNICAMP - Campinas (SP) - Brasil.

2 Pós-graduando do Departamento de Tocoginecologia da Faculdade de Ciências Médicas da Universidade Estadual de Campinas - UNICAMP - Campinas (SP), Brasil.

3 Professor-Titular, Chefe do Departamento de Tocoginecologia da Faculdade de Ciências Médicas da Universidade Estadual de Campinas - UNICAMP - Campinas (SP), Brasil.

Correspondência: Divisão de Obstetrícia/CAISM/UNICAMP

Rua Alexander Fleming, 101 - Cidade Universitária - 13083-881 - Campinas - SP - e-mail: elianaa@unicamp.br 


\section{Introdução}

Métodos seguros de prevenção de gravidez são muito importantes para mulheres soropositivas para o virus da imunodeficiência humana (HIV) ou sob risco de infecção pelo vírus, mas há pouca informação disponivel para avaliar os riscos potenciais da contracepção hormonal nessas mulheres ${ }^{1}$. Os possiveis mecanismos biológicos pelos quais a contracepção hormonal facilitaria a transmissão do HIV incluem² a) aumento da incidência de ectrópio associado ao uso de contraceptivos orais; b) aumento da infecção cervical por clamídia; c) mudança na resposta imunológica humoral e mediada por células, associadas ao uso de esteróides exógenos; d) influência direta de hormônios sexuais na virulência do HIV; e) adelgaçamento do epitélio vaginal e sangramento uterino anormal, associados ao uso de progestógenos injetáveis. Há, ainda, a preocupação de que a gravidez e os contraceptivos possam afetar a vulnerabilidade ao HIV e, entre as mulheres HIV-positivas, a transmissibilidade e a progressão da doença ${ }^{3}$.

Este artigo visa revisar os conhecimentos e recomendações de uso de contraceptivos, particularmente de composição hormonal, concomitante ao uso de antiretrovirais, no cuidado à mulher infectada pelo HIV. Para tanto, foi realizada uma busca no Medline, utilizando-se os termos " $H I V$, antiretroviral, contraception, hormonal contraceptive, AIDS". Também foram revistas as recomendações da Organização Mundial da Saúde (OMS) sobre a indicação dos contraceptivos e os consensos terapêuticos para infecção pelo HIV publicados pelo Ministério da Saúde do Brasil.

\section{Risco de infecção e contracepção hormonal}

De um conjunto de estudos com animais epidemiológicos e de intervenção emergem possíveis mecanismos pelos quais os hormônios podem afetar a aquisição do HIV ${ }^{4}$. Altos níveis de progesterona ou a administração de acetato de medroxiprogesterona de depósito (AMPD), mesmo em baixas doses, levam ao adelgaçamento epitelial em macacos, mas o AMPD não causa adelgaçamento clinicamente significativo em mulheres ${ }^{5}$. Não se confirmou se a fase lútea é um momento de risco aumentado de aquisição do $\mathrm{HIV}^{4}$.

Estudos em populações de alto risco, como prostitutas, que têm múltiplos parceiros e podem ser expostas a múltiplas variantes do HIV, indicam que haveria um risco aumentado de infecção com o uso de contraceptivos hormonais. Entretanto, é possível que, para outros grupos de risco, como mulheres monogâmicas, o uso de contraceptivos hormonais não altere significativamente o risco de aquisição do $\mathrm{HIV}^{6}$. Um estudo prospectivo, por exemplo, concluiu que o uso de contracepção hormonal não estava associado com a aquisição do HIV após ajuste de fatores de confusão ${ }^{7}$. Pode ser que a medida da exposição ao contraceptivo e do elo temporal entre a contracepção e a infecção pelo HIV, ao variar entre os diversos estudos, explique as diferenças e as conclusões opostas observadas. Os resultados distintos podem, também, refletir diferenças reais entre os grupos estudados ${ }^{6}$.

Além disso, há carência de dados sobre a interação entre contracepção hormonal e HIV em subgrupos específicos, particularmente em adolescentes, que parecem ser especialmente vulneráveis à infecção. Também falta informação sobre o risco de aquisição do HIV entre usuárias de outros métodos contraceptivos (implantes, dispositivos intra-uterinos, métodos de barreira além do preservativo). Como não há consenso sobre o efeito da contracepção hormonal e o risco de a mulher adquirir infecção por HIV, a recomendação atual após o encontro internacional sobre contracepção hormonal e HIV, coordenado pela OMS, não restringe o uso de contraceptivos orais e AMPD ${ }^{8}$.

\section{A contracepção hormonal em mulheres infectadas pelo HIV}

Também interessa compreender a influência da contracepção hormonal em mulheres infectadas pelo HIV, incluindo efeitos colaterais, distúrbios menstruais, progressão da doença e interações com terapias anti-retrovirais (TARVs). Essas dúvidas são particularmente importantes no Brasil, onde há acesso universal à TARV, disponibilizada pelo serviço público desde 1991.

Em linhas gerais, a TARV é composta por combinações de fármacos pertencentes à classe dos inibidores da transcriptase reversa, incluindo os análogos nucleosídicos - ITRNs (zidovudina, didanosina, estavudina, lamivudina, zalcitabina e abacavir), os análogos não-nucleosídicos - ITRNN (efavirenz, nevirapina e delavirdina) e o análogo nucleotídico tenofovir; à classe dos inibidores de protease (amprenavir, atazanavir, indinavir, lopinavir, ritonavir, nelfinavir, saquinavir) ou à classe dos inibidores de fusão (enfuvirtida). Com exceção da gravidez, a terapia inicial deve incluir ao menos três drogas, dois ITRN associados a um ITRNN ou a um inibidor de protease ${ }^{9}$. No mais recente consenso brasileiro sobre TARV, os esquemas compostos por zidovudina, lamivudina e efavirenz ou zidovudina, lamivudina e atazanavir ou 
lopinavir, associados a ritonavir têm sido adotados como primeira opção ${ }^{10}$. Embora o uso de TARV pela mulher e pelo recém-nascido, a cesariana eletiva e a recomendação de não-amamentação tenham contribuído para uma diminuição acentuada da transmissão vertical do $\mathrm{HIV}^{11}$, a mulher HIV-positiva usando TARV que não deseja engravidar precisa ter disponiveis métodos seguros de contracepção.

Sabe-se que o uso consistente do preservativo masculino reduz a incidência da infecção pelo HIV em $80 \%$, mas a taxa de falha na prevenção de gravidez é de $15 \%$ no primeiro ano de uso típico ${ }^{12}$. Essa taxa chega a $21 \%$ com o preservativo feminino ${ }^{13}$. Torna-se necessária, portanto, a associação de um contraceptivo cuja taxa de falha em uso típico seja baixa.

A contracepção hormonal em mulheres HIV-positivas está associada a um risco aumentado de cervicite e infecção cervical por clamídia. Como essas infecções aumentam a eliminação do HIV nas secreções cervicais, essas mulheres estão sob risco aumentado de transmitir o HIV aos parceiros sexuais ${ }^{14}$. Além disso, as infecções sexualmente transmissiveis aumentam a carga viral do HIV, o que poderia causar um curso mais rápido da doença relacionada ao virus ${ }^{14}$.

Os anticoncepcionais hormonais estão entre os contraceptivos mais eficazes e não interferem na resposta imunológica (avaliada pela contagem de células $\mathrm{CD} 4+$ ) e virológica (avaliada pela carga viral) à TARV ${ }^{15}$, mas, por compartilhar vias metabólicas com os ARVs, há dúvidas quanto à possivel interação medicamentosa. O AMPD, usado por grande proporção de mulheres, torna-se especialmente importante como alvo de investigação de potencial interação com a TARV ${ }^{16}$. O progestógeno levonorgestrel (LNG), administrado através do sistema intra-uterino liberador, mostrou-se seguro num pequeno grupo de mulheres infectadas pelo HIV. Não houve aumento da eliminação vaginal do HIV e houve redução da perda sanguínea, com aumento da hemoglobina ${ }^{17}$.

\section{As interações entre hormônios contraceptivos}

\section{e antiretrovirais}

As interações medicamentosas podem ser farmacocinéticas (alterações da absorção, distribuição ou eliminação de um fármaco por outro) ou farmacodinâmicas (por exemplo, interações nos receptores dos fármacos) e o resultado da interação farmacocinética pode ser um aumento ou uma redução da concentração do fármaco no local de ação ${ }^{18}$. A biotransformação, uma das etapas da farmacocinética, é afetada por polimorfismos, uso concomitante de outras drogas, exposição a poluentes ambientais e substâncias químicas industriais, doença, estado clínico e idade e, embora as interações farmacocinéticas possam causar alterações na absorção, na ligação à proteína e na excreção urinária, o efeito na biotransformação costuma ser o mais acentuado ${ }^{19}$.

A superfamília de enzimas do citocromo P450 é a principal responsável pelas reações de biotransformação de fármacos. Entre essas enzimas, a CYP3A4 e a CYP3A5 são as mais abundantemente expressas no figado e no trato gastrointestinal humanos e são responsáveis pelo metabolismo de mais de 120 drogas diferentes, como acetaminofeno, codeína, diazepam, eritromicina, lidocaina e lovastatina $^{20}$. A exposição a certos fármacos e poluentes ambientais associa-se a aumento da sintese da proteína do citocromo P450, e essa indução enzimática determina maior velocidade de biotransformação e reduções correspondentes na disponibilidade do fármaco original ${ }^{19}$. A indução enzimática também pode associar-se ao aumento da toxicidade no caso de fármacos metabolizados em formas reacionais. Em alguns casos, determinado composto pode induzir a biotransformação de outros e o seu próprio metabolismo. A inibição enzimática, por sua vez, resulta em niveis elevados do fármaco original, efeitos farmacológicos prolongados e maior incidência de toxicidade da droga. Exemplo de interação medicamentosa é a indução potente de CYP3A4 intestinal e hepática pela rifamicina, que determina aumento significativo na depuração de corticosteróides, diazepam, anticoncepcionais orais etc. Além disso, as diferenças genéticas (polimorfismo genético) na capacidade das pessoas de metabolizar um fármaco através de determinada via são as responsáveis pelas grandes diferenças interindividuais na biotransformação observada numa população. As diferenças fenotípicas na quantidade da droga excretada através de uma via polimorficamente controlada levam à classificação dos individuos em metabolizadores rápidos ou lentos ${ }^{19}$.

Assim como os inibidores de protease, todos os ITRNN são metabolizados pelo sistema enzimático do citocromo $\mathrm{P} 450$, o que os torna propensos a interações medicamentosas clinicamente significativas $^{20}$. O etinilestradiol (EE), principal componente estrogênico dos contraceptivos orais combinados, é metabolizado por hidroxilação mediada por CYP3A e submete-se à conjugação com ácido glicurônico e sulfato ${ }^{21}$. O acetato de medroxiprogesterona também é metabolizado pelo citocromo P450 CYP3A $4{ }^{22}$. Sabe-se que o ritonavir reduz a concentração plasmática de EE através do aumento da depuração por indução enzimática, fato que sugere o uso de métodos anticoncepcionais alternativos 
Tabela 1 - Interações farmacocinéticas entre contraceptivos orais combinados e anti-retrovirais ${ }^{13}$.

\begin{tabular}{|c|c|c|}
\hline Antiretrovirais (ARV) & Níveis de esteróides contraceptivos & Níveis de ARVs \\
\hline \multicolumn{3}{|l|}{ Inibidores de protease } \\
\hline Lopinavir/ritonavir & $\downarrow$ & Sem dados \\
\hline Atazanavir & $\uparrow$ & Sem dados \\
\hline Nelfinavir & $\downarrow$ & Sem dados \\
\hline Ritonavir & $\downarrow$ & Sem dados \\
\hline Amprenavir & $\uparrow$ & $\downarrow$ \\
\hline Indinavir & $\uparrow$ & Sem dados \\
\hline Saquinavir & Sem dados & Sem alterações \\
\hline \multicolumn{3}{|c|}{ Inibidores da transcriptase reversa não-nucleosídicos } \\
\hline Efavirenz & 个 & Sem alterações \\
\hline Nevirapina & $\downarrow$ & Sem alterações \\
\hline Delavirdina & $? \uparrow$ & Sem dados \\
\hline
\end{tabular}

Fonte: World Health Organization. Medical eligibility criteria for contraceptive use. 3rd ed. Geneva: WHO; 2004

$\boldsymbol{\uparrow}=$ aumento; $\boldsymbol{\Downarrow}=$ diminuição.

quando o ritonavir estiver sendo administrado com $\mathrm{o} \mathrm{EE}^{23}$. A nevirapina interfere na farmacocinética do EE e da noretisterona, reduzindo a área sob a curva da concentração plasmática de ambas as drogas; sugeriu-se que os contraceptivos orais não deveriam ser o método primário de contracepção das mulheres tratadas com neviparina ${ }^{24}$. Não há dados específicos sobre interações entre o regime de contracepção de emergência com o progestógeno LNG, usado oralmente como contraceptivo de emergência no caso de falha do uso do preservativo, e as drogas antiretrovirais ${ }^{25}$.

A contracepção combinada oral, por sua vez, não altera a farmacocinética do saquinavir em mulheres ${ }^{26}$. Além desses estudos farmacocinéticos, pouco se sabe sobre as interações medicamentosas envolvidas entre contraceptivos hormonais e ARVs, pois os dados disponiveis limitam-se àqueles obtidos pela indústria farmacêutica durante o processo de aprovação das drogas ${ }^{27}$. Poucos dados de estudos pequenos, nem sempre publicados, sugerem que a farmacocinética de uma dose única de contraceptivo oral combinado pode ser alterada de forma diversa segundo as várias terapias antiretrovirais, com direções opostas dentro da mesma categoria de drogas. Os caros estudos de farmacocinética dos antiretrovirais com uso de hormônios e vice-versa não acompanham o rápido avanço da terapia e suas variadas combinações. Os critérios de elegibilidade médica para o uso de contraceptivos da OMS resumem dados pouco conclusivos dos efeitos dos ARVs nos níveis de esteróides contraceptivos e destes sobre os niveis de ARVs (Tabela 1).

\section{Conclusões}

Não é possivel, baseando-se em evidências, elaborar recomendações para contracepção hormonal de mulheres portadoras do HIV sob TARV. Os infectologistas e os ginecologistas que dão assistência a essas mulheres devem estar atentos às interações potenciais que possam representar aumento de efeitos adversos, individualizando a orientação sobre os esteróides contraceptivos, suas doses e vias de administração, de acordo com a TARV em uso.

\section{Referências}

1. Baeten JM, Lavreys L, Sagar M, Kreiss JK, Richardson BA, Chohan B, et al. Effect of contraceptive methods on natural history of HIV: studies from the Mombasa cohort. J Acquir Immune Defic Syndr. 2005; 38 Suppl 1:S18-21.

2. Morrison CS, Richardson BA, Celentano DD, Chipato T, Mmiro F, Mugerwa R, et al. Prospective clinical trials designed to assess the use of hormonal contraceptives and risk of HIV acquisition. J Acquir Immune Defic Syndr. 2005; 38 Suppl 1:S17-8.

3. Allen S. Why is fertility an issue for HIV-infected and at-risk women? J Acquir Immune Defic Syndr. 2005; 38 Suppl 1:S1-3.

4. Mauck C. Overview of why hormones may be an issue. J Acquir Immune Defic Syndr. 2005; 38 Suppl 1:S11-2.

5. Bahamondes L, Trevisan M, Andrade L, Marchi NM, Castro S, Díaz J, et al. The effect upon the human 
vaginal histology of the long-term use of the injectable contraceptive Depo-Provera ${ }^{\circledR}$. Contraception. 2000; 62(1):23-7.

6. Martin HL Jr, Richardson BA, Mandaliya K, Achola JO, Overbaugh J, Kreiss JK. The early work on hormonal contraceptive use and HIV acquisition. J Acquir Immune Defic Syndr. 2005; 38 Suppl 1:S12-4.

7. Kiddugavu M, Makumbi F, Wawer MJ, Serwadda D, Sewankambo NK, Wabwire-Mangen F, et al. Hormonal contraceptive use and HIV-1 infection in a population-based cohort in Rakai, Uganda. AIDS. 2003; 17(2):233-40.

8. World Health Organization. Hormonal contraception and HIV: Science and Policy. Africa Regional Meeting; 2005 Sep 19-21; Nairobi, Africa; 2005.

9. Robbins GK, De Gruttola V, Shafer RW, Smeaton LM, Snyder SW, Pettinelli C, et al. Comparison of sequential three-drug regimens as initial therapy for HIV-1 infection. N Engl J Med. 2003; 349(24):2293-303.

10.Ministério da Saúde. Recomendações para terapia anti-retroviral em adultos e adolescentes infectados pelo HIV. Brasília; 2006.

11.Ministério da Saúde. Recomendações para profilaxia da transmissão vertical do HIV e terapia antiretroviral em gestantes. Brasília; 2006. 81p.

12.Weller S, Davis K. Condom effectiveness in reducing heterosexual HIV transmission Cochrane Database Syst Rev. 2005;(2):CD003255.

13.World Health Organization. Medical eligibility criteria for contraceptive use. $3^{\text {rd }}$ ed. Geneva: WHO; 2004.

14.Lavreys L, Chohan V, Overbaugh J, Hassan W, McClelland RS, Kreiss J, et al. Hormonal contraception and risk of cervical infections among HIV-1-seropositive Kenyan women. AIDS. 2004; 18(16):2179-84.

15. Chu JH, Gange SJ, Anastos K, Minkoff H, Cejtin H, Bacon $\mathrm{M}$, et al. Hormonal contraceptive use and the effectiveness of highly active antiretroviral therapy. Am J Epidemiol. 2005; 161(9):881-90.

16.Clark RA, Theall KP. Trends and correlates of hormonal contraception use among HIV-infected women. J Acquir Immune Defic Syndr. 2004; 36(4):986-8.
17. Heikinheimo O, Lehtovirta P, Suni J, Paavonen J. The levonorgestrel-releasing intrauterine system (LNG-IUS) in HIV-infected women--effects on bleeding patterns, ovarian function and genital shedding of HIV. Hum Reprod. 2006; 21(11):2857-61.

18. Nies AS, Spielberg SP. Princípios da terapêtica. In: Hardman JG, Limbird LE, Molinoff PB, Ruddon RW, Gilman AG. Goodman \& Gilman as bases farmacológicas da terapêutica. $9^{\mathrm{a}}$ ed. New York: McGraw-Hill; 1996. p. 31-44.

19.Benet LZ, Kroetz DL, Sheiner LB. Farmacocinética A dinâmica da absorção, distribuição e eliminação dos fármacos. In: Hardman JG, Limbird LE, Molinoff PB, Ruddon RW, Gilman AG. Goodman \& Gilman as bases farmacológicas da terapêutica. $9^{\mathrm{a}} \mathrm{ed}$. New York: McGraw-Hill; 1996. p. 3-20.

20. SmithPF, DiCenzo R, Morse GD. Clinical pharmacokinetics of non-nucleoside reverse transcriptase inhibitors. Clin Pharmacokinet. 2001; 40(12):893-905.

21. Nebert DW, Russell DW. Clinical importance of the cytochromes P450. Lancet. 2002; 360(9340):1155-62.

22.Kobayashi K, Mimura N, Fujii H, Minami H, Sasaki $\mathrm{Y}$, Shimada N, et al. Role of human cytochrome P450 3A4 in metabolism of medroxyprogesterone acetate. Clin Cancer Res. 2000; 6(8):3297-303.

23. Ouellet D, Hsu A, Qian J, Locke CS, Eason CJ, Cavanaugh $\mathrm{JH}$, et al. Effect of ritonavir on the pharmacokinetics of ethinyl oestradiol in healthy female volunteers. Br J Clin Pharmacol. 1998; 46(2):111-6.

24. Mildvan D, Yarrish R, Marshak A, Hutman HW, McDonough M, Lamson M, et al. Pharmacokinetic interaction between nevirapine and ethinyl estradiol/ norethindrone when administered concurrently to HIV-infected women. J Acquir Immune Defic Syndr. 2002; 29(5):471-7.

25. Heard I. Data from the French cohort studies and framing the French contraceptive guidelines. J Acquir Immune Defic Syndr. 2005; 38 Suppl 1:S29-31.

26.Fröhlich M, Burhenne J, Martin-Facklam M, Weiss $\mathrm{J}$, von Wolff M, Strowitzki T, et al. Oral contraception does not alter single dose saquinavir phamacokinetics in women. Br J Clin Pharmacol. 2004; 57(3):244-52.

27. Catanzaro LM, Morse GD. Data from clinical trials. J Acquir Immune Defic Syndr. 2005; 38 Suppl 1:S26-9. 